\title{
The mathematical constraint of the counterexample of Goldbach's strong conjecture
}

Ahmad Hazaymeh, Ministry of Education, Irbid, Jordan ahmad.hazaymeh@ses.yu.edu.jo

Dr. Khaled Hazaymeh, Yarmouk University, Jordan. khazaymeh@yu.edu.jo

Sukaina Hazaymeh, Yarmouk University, Jordan.

sukaina.hazaymeh@ses.yu.edu.jo

\begin{abstract}
In this paper, we have demonstrated a proof that the Counterexample of Goldbach's strong conjecture is impossible in two steps: First, we reformulated Goldbach's strong conjecture using the subtraction connotation. Second: the mathematical Constraint that must be fulfilled in any even number has been deduced to be that even number a Counterexample of Goldbach's strong conjecture. Then we demonstrated that any counterexample would fulfill this mathematical Constraint. It will either contradict the theorem of infinite prime numbers or contradict the Prime Number Theorem. Therefore, the logical conclusion is that there is no counterexample to Goldbach's strong conjecture. With the absence of a counter-example, Goldbach's strong conjecture would be a true conjecture.
\end{abstract}

\section{Introduction}

Goldbach formulated his conjecture in connotation of addition, which made conjecture difficult to prove for a long time. Surprisingly, proof of conjecture will be handy, once we reformulated Goldbach's strong conjecture using the subtraction connotation. We think that, if the conjecture was reformulated using the subtraction connotation, the conjecture would have been proven from a long ago, but for some reason, no one did that.

\section{Goldbach's strong conjecture using the subtraction connotation}

Goldbach's strong conjecture states: "Every even integer greater than 4 can be expressed as the sum of two odd primes"

First, we will rephrase Goldbach's conjecture using a subtraction connotation; we will call this new formulation as "Goldbach's strong conjecture in connotation of subtraction" to distinguish it from "Goldbach's strong conjecture in connotation of addition".

The new proposed formulation the Goldbach's strong conjecture states that "Every even number greater than 4 , if we subtract from it all the prime odd numbers smaller than it (each prime 
number separately) then one of the outputs of the subtraction will be a prime odd number. This will happen at least once. "

Example 1: An example of an even number, where we subtract all smaller prime odd than it.

\begin{tabular}{|l|}
\hline $20-3=17$ \\
\hline $20-5=15$ \\
\hline $20-7=13$ \\
\hline $20-11=9$ \\
\hline $20-13=7$ \\
\hline $20-17=3$ \\
\hline $20-19=1$ \\
\hline
\end{tabular}

Example 2: If the result of subtracting a prime number from an even number equals to a prime number, then the sum of the two prime numbers will equal the same even number.

$$
\begin{array}{|l|}
\hline 3+17=20 \\
\hline 7+13=20 \\
\hline
\end{array}
$$

\section{Three possibilities}

Question: If we subtract from every even number all the odd prime numbers smaller than it does, what are the possibilities of the subtraction results?

Answer: three possibilities.

Probability number 1: The subtraction results will be either the odd prime numbers or the odd multiple numbers. (See example 1). If this possibility achieved, then Goldbach's strong conjecture will be true.

Probability number 2: The subtraction results will be only the prime numbers. If this possibility achieved, then Goldbach's strong conjecture will be true.

Probability number 3: The subtraction results will be the odd multiple numbers. If this possibility achieved, then Goldbach's strong conjecture will be false.

\section{First assumption}

The conjecture has been shown to hold for all integers less than $4 \times 10^{18}$ but remains unproven despite considerable effort. A question would be mentioned:

"Is it possible that there is an even number if we subtract from it all the smaller prime odd numbers than it, (each prime odd number separately), then the result of all subtraction outputs will be only multiple odd numbers?" 


\subsection{First contradiction}

Because the existence of the counterexample is possible, we should prove one of the following two possibilities:

Either that this a counter-example is existed, which then will prove that Goldbach's strong conjecture is false. Either it is not existed, which will prove that Goldbach's strong conjecture is true. In order for the counter-example to be existed, the following must be achieved:

First: The prime numbers set must be a finite set. We will assume that the set of finite prime numbers are $(2,3,5,7, \ldots, Z)$ where the number $(Z)$ is the last prime number.

Second: We will multiply all prime numbers together

$$
(2 \times 3 \times 5 \times 7 \times \ldots \times Z) .
$$

Before we move to (third), we will explain the following example:

A. For example, we will assume we have a subset of $(2,3,5,7)$.

B. The multiplying of subset will be (210).

C. If we subtract from the number (210) all the odd prime numbers of subset, which are $(3,5,7)$. The result of each subtraction process will be an odd number of multiples of the prime number that we subtract from the even number, as shown in Table 1.

Table 1: The table shows that the result of each subtraction process will be an odd number of multiples of the prime number that we subtract from the even number.

\begin{tabular}{|l|}
\hline$[3(2 \times 5 \times 7)-3]=x$ \\
{$[(3 \times 70)-3]=(3 \times 69)$} \\
{$[210-3]=207$} \\
$\quad$ Where $(207)$ is an odd number of multiples of the prime number 3 \\
\hline$[5(2 \times 3 \times 7)-5]=x$ \\
{$[(5 \times 42)-5]=(5 \times 41)$} \\
{$[210-5]=205$} \\
$\quad$ Where $(205)$ is an odd number of multiples of the prime number 5 \\
\hline$[7(2 \times 3 \times 5)-7]=x$ \\
{$[(7 \times 30)-7]=(7 \times 29)$} \\
{$[210-7]=203$} \\
Where $(203)$ is an odd number of multiples of the prime number 7 \\
\hline
\end{tabular}


Third: (remember the previous example when reading "third"). After we multiply all the prime numbers together.

$$
(2 \times 3 \times 5 \times 7 \times \ldots \times Z=k)
$$

The result of multiplication will be an even number because we multiplied all numbers to the number 2. We will call this assumed even number as $(k)$. If we subtract from this assumed even number $(k)$ all the smaller odd prime numbers than it. The result of each subtraction process will be an odd number of multiples of the prime number that we subtract from the assumed even number, as shown in Table 2:

Table 2: The table shows that the result of each subtraction process will be an odd number of multiples of the prime number that we subtract from the assumed even number.

$$
\begin{aligned}
& (k)-(3)=(k-3) \\
& \text { Where number }(k-3) \text { is an odd number of multiples of the prime number (3) } \\
& (k)-(5)=(k-5) \\
& \text { Where number }(k-5) \text { is an odd number of multiples of the prime number (5) } \\
& (k)-(7)=(k-7) \\
& \text { Where number }(k-7) \text { is an odd number of multiples of the prime number (7) } \\
& (k)-(Z)=(k-Z) \\
& \text { Where number }(k-Z) \text { is an odd number of multiples of the prime number }(Z)
\end{aligned}
$$

Then we will find that the assumed even number $(k)$ has fulfilled the previous assumption, which assumes the possibility that there is an even number that if we subtract from it all the smaller prime odd numbers than it (each prime odd number separately), then the result of all subtraction outputs will be only multiple odd numbers." This proves that the Goldbach's strong conjecture is false.

However, remember...

The Counterexample (the assumed even number " $k$ ") was resulted from a false premise; it was the assumption that the prime numbers are a finite set. However, the fact is that the set of prime numbers is an infinite set (see, for example, Euclid's theorem), this fact will negate the existence of that Counterexample (The assumed even number " $k$ "). Because either the Counterexample is existed or the prime numbers are an infinite set, but both probabilities will not be true together, because this will be a contradiction. 
1. $\left(p_{1} \times \mathrm{p}_{2} \times \mathrm{p}_{3} \times \mathrm{p}_{4} \times \ldots \times Z=k\right) \vee\left(p_{1} \times \mathrm{p}_{2} \times \mathrm{p}_{3} \times \mathrm{p}_{4} \times \ldots\right)$

2. $\left(p_{1} \times \mathrm{p}_{2} \times \mathrm{p}_{3} \times \mathrm{p}_{4} \times \ldots\right) \rightarrow \neg k$

3. $(\neg k \rightarrow$ Goldbach is valid $)$

In other words, if the counterexample of Goldbach's strong conjecture (the assumed even number " $k$ ") does not exist, then Goldbach's strong conjecture in subtraction connotation will be a valid conjecture. Moreover, if Goldbach's strong conjecture in subtraction connotation is valid, and then Goldbach's strong conjecture in addition connotation will be also valid conjecture.

\section{Second assumption}

Well, if the counterexample (the assumed even number " $k$ ") contradicts the theorem of infinite prime numbers because of the Counterexample (the assumed even number " $k$ ") was resulted from a false premise, Is it possible that there is an even number actually if we subtract from it all the smaller prime odd numbers than it does, (each prime odd number separately), then the result of all subtraction outputs will be only multiple odd numbers.

\subsection{Generalization of Constraint (Counterexample " $l$ ")}

Remember, the assumed even number $(k)$ has become a Counterexample because it is a number that results from multiplying of entire prime numbers that are smaller than the assumed even number $(\mathrm{k})$ :

$$
(2 \times 3 \times 5 \times 7 \times \ldots \times z=k)
$$

Therefore, any even number to be a Counterexample of Goldbach's strong conjecture, then that number must fulfill the Constraint that the supposed even number $(k)$ fulfills, the mathematical Constraint of Counterexample of Goldbach's strong conjecture is:

"To achieve a counterexample of Goldbach's strong conjecture, the product of multiplying all the prime numbers smaller than the even number must be equal to the value of the even number itself". We will call the even number that fulfills this Constraint $(l)$.

$$
\begin{aligned}
& \text { 1. }\left(p_{1}, \mathrm{p}_{2}, \mathrm{p}_{3}, \mathrm{p}_{4}, \ldots, \mathrm{m}\right)<l \\
& \text { 2. }\left(p_{1} \times \mathrm{p}_{2} \times \mathrm{p}_{3} \times \mathrm{p}_{4} \times \ldots \times \mathrm{m}=l\right)
\end{aligned}
$$

$l$ : Actual even number.

$m$ : The last prime number is smaller than the even number $(l)$.

\section{2 second contradiction (illogical gap)}

We can say intuitively that it seems impossible for the product of all prime numbers that are smaller than any even number to be equal to the value of the even number. 
Table 3: Even numbers where the product of all the prime numbers smaller than the even number do not equal the value of the even number.(That is, the even numbers do not fulfill the Constraint that the assumed number $(k)$ was fulfilled).

\begin{tabular}{|c|c|}
\hline $\begin{array}{c}\text { Even } \\
\text { numbers }\end{array}$ & Product of prime numbers smaller than even number $\neq$ even number \\
\hline 6 & $2 \times 3 \times 5 \neq 6$ \\
\hline 10.8 & $2 \times 3 \times 5 \times 7 \neq 8$ or 10 \\
\hline 12 & $2 \times 3 \times 5 \times 7 \times 11 \neq 12$ \\
\hline 14,16 & $2 \times 3 \times 5 \times 7 \times 11 \times 13 \neq 14$ or16 \\
\hline
\end{tabular}

However, is any an even number having an actual presence equal to the product of all the prime numbers smaller than it, is it actually possible?

Answer: No, because the existence of this type of counterexample will create an illogical gap. A very huge gap and devoid of any prime number between the number $(m)$ which is the last prime number smaller than the even number, and the even number $(l)$ which is the product of multiplying all the prime numbers smaller than it. The existence of this very huge gap and devoid of any prime number contradict with the Prime Number Theorem.

$$
\begin{aligned}
& \text { 1. }\left(p_{1}, \mathrm{p}_{2}, \mathrm{p}_{3}, \mathrm{p}_{4}, \ldots, \mathrm{m}\right)<l \\
& \text { 2. }\left(p_{1} \times \mathrm{p}_{2} \times \mathrm{p}_{3} \times \mathrm{p}_{4} \times \ldots \times m=l\right) \\
& \text { 3. }(m<\{\}<l)
\end{aligned}
$$

$l$ : Actual even number.

$m$ : The last prime number is smaller than the even number $(l)$.

\{\}$:$ A very huge gap, devoid of any prime.

Example: If we assume that, the even number $(6,469,693,230)$ which is the product of multiplying the first ten prime numbers only.

$$
(2 \times 3 \times 5 \times 7 \times 11 \times 13 \times 17 \times 19 \times 23 \times 29=6,469,693,230)
$$

Assuming this even number is Counterexample of Goldbach's strong conjecture, it will result in the becoming the prime number $(29)$ is the number $(m)$, and That is, it will be the last prime number smaller than the even number. Therefore, it will create a very huge gap between the number (29) and the even number $(6,469,693,230)$, and it will be a very huge gap, devoid of any prime number.

$$
(29<\{\quad\}<6,469,693,230)
$$

$\{$ \}: A very huge gap, devoid of any prime. 
The existence of this very huge gap contradicts with the Prime Number Theorem. The logical result is that the even number that is the product of all the prime numbers smaller than it is a number that does not exist in the natural numbers. Thus, we do not find huge gaps between the prime numbers.

\section{Conclusion}

Any counterexample of Goldbach's strong conjecture is either it would contradict the infinite theorem of prime numbers, either it would contradict The Prime Number Theorem. Therefore, the logical conclusion is that there is no counterexample for Goldbach's conjecture. With the absence of the counterexample, Goldbach's strong conjecture will be valid conjecture.

Note: We thank all professors and colleagues whom we discussed the idea of this proof, as their discussions had great value in the final wording for this paper. 